\title{
Isospin Violation and the Magnetic Moment of the Muon
}

\author{
Vincenzo Cirigliano*, Gerhard Ecker, Helmut Neufeld ${ }^{\dagger}$ \\ Institut für Theoretische Physik, Universität Wien \\ Boltzmanngasse 5, A-1090 Vienna, Austria \\ E-mail: ivincenzo@thp.univie.ac. at'
}

ABstract: We calculate the leading isospin-violating and electromagnetic corrections for the decay $\tau^{-} \rightarrow \pi^{0} \pi^{-} \nu_{\tau}$ at low energies. The corrections are small but relevant for the inclusion of $\tau$ decay data in the determination of hadronic vacuum polarization especially for the anomalous magnetic moment of the muon. This contribution is based on Ref. [1] $\left.]_{1}^{1}\right]$

\section{Introduction}

The leading hadronic contribution to the anomalous magnetic moment of the muon $a_{\mu}=$ $\left(g_{\mu}-2\right) / 2$ is given by the hadronic vacuum polarization [2]2]:

$$
a_{\mu}^{\mathrm{vacpol}}=\frac{1}{4 \pi^{3}} \int_{4 M_{\pi}^{2}}^{\infty} d t K(t) \sigma_{e^{+} e^{-} \rightarrow \text { hadrons }}^{0}(t)
$$

where $K(t)$ is a smooth kernel concentrated at low energies, and $\sigma_{e^{+} e^{-} \rightarrow \text { hadrons }}^{0}$ denotes the "pure" hadronic cross section with QED corrections removed. The low-energy structure of hadronic vacuum polarization is especially important. In fact, about $70 \%$ of $a_{\mu}^{\text {vacpol }}$ is due to the two-pion intermediate state for $4 M_{\pi}^{2} \leq t \leq 0.8 \mathrm{GeV}^{2}$ (see, e.g., Ref. [iji]). A precision of $1 \%$ has been achieved in the calculation of $a_{\mu}^{\mathrm{vacpol}}$ by including the more accurate $\tau$ decay data $[-15]$ in addition to $\sigma\left(e^{+} e^{-} \rightarrow\right.$ hadrons $)$ data. This is possible because of a CVC relation between electromagnetic and weak form factors in the isospin limit. However, both the aforementioned theoretical accuracy and the new high-precision experiment at Brookhaven [i $[\overline{6}]$ warrant a closer investigation of isospin violation, due to both the light quark mass difference and electromagnetism (EM). We concentrate in this work on isospin violation in the reactions $\tau^{-} \rightarrow \pi^{0} \pi^{-} \nu_{\tau}$ and $e^{+} e^{-} \rightarrow \pi^{+} \pi^{-}$at low energies. Chiral perturbation theory (CHPT) [i], is is the framework where such corrections can

${ }^{*}$ Speaker.

${ }^{\dagger}$ Work supported in part by TMR, EC-Contract No. ERBFMRX-CT980169 (EURODAФNE). 
reliably be calculated for the standard model in a systematic low-energy expansion. More specifically, we calculate the leading corrections of both $O\left[\left(m_{u}-m_{d}\right) p^{2}\right]$ and $O\left(e^{2} p^{2}\right)$ for the CVC relation between the two-pion (vector) form factors in the two processes. For a more detailed presentation and a more complete bibliography we refer to Ref. [iin.

Let us define the problem more precisely. For the two-pion final state, the bare $e^{+} e^{-}$ cross section and the $\tau^{-} \rightarrow \pi^{0} \pi^{-} \nu_{\tau}$ decay distribution take the form:

$$
\begin{aligned}
\sigma_{e^{+} e^{-} \rightarrow \pi^{+} \pi^{-}}^{0}(t) & =\frac{\pi \alpha^{2}}{3 t} \beta_{\pi^{+} \pi^{-}}^{3}(t)\left|F_{V}(t)\right|^{2} \\
\frac{d \Gamma\left(\tau^{-} \rightarrow \pi^{0} \pi^{-} \nu_{\tau}\right)}{d t} & =\Gamma_{e} \mathcal{K}_{1}(t) \beta_{\pi^{0} \pi^{-}}^{3}(t)\left|f_{+}(t)\right|^{2} S_{\mathrm{EW}} G_{\mathrm{EM}}(t),
\end{aligned}
$$

where

$$
\Gamma_{e}=\frac{G_{F}^{2} m_{\tau}^{5}}{192 \pi^{3}}, \quad \mathcal{K}_{1}(t)=\frac{\left|V_{u d}\right|^{2}}{2 m_{\tau}^{2}}\left(1-\frac{t}{m_{\tau}^{2}}\right)^{2}\left(1+\frac{2 t}{m_{\tau}^{2}}\right) .
$$

Here $t$ is the hadronic invariant mass; $\beta_{\pi^{+} \pi^{-}}(t)$ and $\beta_{\pi^{0} \pi^{-}}(t)$ are the center of mass pion velocities for the two processes; $F_{V}(t)$ and $f_{+}(t)$ are the EM and weak vector form factors of the pion. In the isospin limit $\left(m_{u}=m_{d}\right.$ and $\left.e=0\right)$ we have $M_{\pi^{+}}=M_{\pi^{0}}$ (hence $\left.\beta_{\pi^{+} \pi^{-}}(t)=\beta_{\pi^{0} \pi^{-}}(t)\right), S_{\mathrm{EW}}=G_{\mathrm{EM}}(t)=1$ and $f_{+}(t)=F_{V}(t)$, implying the CVC relation

$$
\sigma_{e^{+} e^{-} \rightarrow \pi^{+} \pi^{-}}^{0, C V C}=\frac{\pi \alpha^{2}}{3 t \mathcal{K}_{1}(t) \Gamma_{e}} \frac{d \Gamma\left(\tau^{-} \rightarrow \pi^{0} \pi^{-} \nu_{\tau}\right)}{d t} .
$$

Including isospin violation, the modified CVC relation reads

$$
\begin{aligned}
\sigma_{e^{+} e^{-} \rightarrow \pi^{+} \pi^{-}}^{0}(t) & =\sigma_{e^{+} e^{-} \rightarrow \pi^{+} \pi^{-}}^{0, C V}(t) \frac{R_{\mathrm{IB}}(t)}{S_{\mathrm{EW}}}, \\
R_{\mathrm{IB}}(t) & =\frac{1}{G_{\mathrm{EM}}(t)} \frac{\beta_{\pi^{+} \pi^{-}}^{3}(t)}{\beta_{\pi^{0} \pi^{-}}^{3}(t)}\left|\frac{F_{V}(t)}{f_{+}(t)}\right|^{2} .
\end{aligned}
$$

The factor $S_{\mathrm{EW}}$ takes into account the dominant short-distance electroweak corrections [9]. To lowest order in $\alpha$, it is given by $S_{\mathrm{EW}}=1+(\alpha / \pi) \log \left(M_{Z}^{2} / m_{\tau}^{2}\right)$, amounting to the numerical value $S_{\mathrm{EW}}=1.0194$. This is consistently used in all present analyses. $R_{\mathrm{IB}}(t)$ involves the long distance QED factor $G_{\mathrm{EM}}(t)$ (which receives both virtual and real photon contributions), the phase space correction factor $\beta_{\pi^{+} \pi^{-}}^{3}(t) / \beta_{\pi^{0} \pi^{-}}^{3}(t)$ [i] $\left.{ }_{1}^{\overline{1}} \overline{0}\right]$, and the ratio of $\mathrm{EM}$ and weak form factors. Working at leading order, the form factor $F_{V}(t)$ needs to be calculated to $O\left[\left(m_{u}-m_{d}\right) p^{2}\right]$ with physical meson masses (but without explicit photonic corrections). $f_{+}(t)$ must be calculated to both $O\left[\left(m_{u}-m_{d}\right) p^{2}\right]$ and $O\left(e^{2} p^{2}\right)$, if $d \Gamma / d t$ is to be extracted from actual $\tau$ decay data.

\section{Anatomy of $R_{\mathrm{IB}}(t): F_{V}, f_{+}, G_{\mathrm{EM}}$}

At first non-trivial order in the low-energy expansion, isospin violation manifests itself in the pion vector form factor $F_{V}(t)$ only in the masses of the particles contained in the loop amplitude:

$$
F_{V}(t)=1+2 H_{\pi^{+} \pi^{-}}(t)+H_{K^{+} K^{-}}(t)
$$


with $[\underline{1}[\underline{1} \overline{1} 1]$

$$
H_{P Q}(t)=\tilde{H}_{P Q}(t, \mu)+\frac{2}{3 F_{\pi}^{2}} t L_{9}^{r}(\mu),
$$

The loop function $\tilde{H}_{P Q}(t)$ encodes the singularities due to the low energy meson propaga-

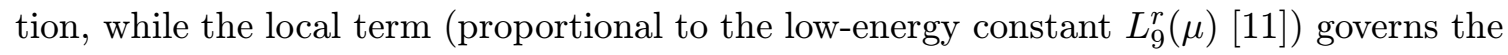
charge radius of the pion and is sensitive to the structure of the theory at higher energies. This specific channel is completely dominated by the $\rho$ resonance. So, leaving the domain of a pure low energy effective theory, we use the prescription of Ref. [i1 $\overline{2}]$ to match the CHPT form factor $(2 . \overline{1})$ of $O\left(p^{4}\right)$ to the resonance region:

$$
\begin{aligned}
F_{V}(t) & =\frac{M_{\rho}^{2}}{M_{\rho}^{2}-t-i M_{\rho} \Gamma_{\rho}(t)} \exp \left[2 \operatorname{Re} \tilde{H}_{\pi^{+} \pi^{-}}(t)+\operatorname{Re} \tilde{H}_{K^{+} K^{-}}(t)\right], \\
\Gamma_{\rho}(t) & =\frac{M_{\rho} t}{96 \pi F_{\pi}^{2}}\left[\beta_{\pi \pi}^{3}(t) \theta\left(t-4 M_{\pi}^{2}\right)+\frac{1}{2} \beta_{K K}^{3}(t) \theta\left(t-4 M_{K}^{2}\right)\right] .
\end{aligned}
$$

For the present case, the charged pion and kaon masses must be inserted in the hadronic off-shell width. The representation $(\overline{2} . \overline{3})$ gives an excellent description of $e^{+} e^{-} \rightarrow \pi^{+} \pi^{-}$ data up to $t \sim 1 \mathrm{GeV}^{2}$ with the single parameter $M_{\rho} \simeq 775 \mathrm{MeV}$, and has the correct low-energy behaviour to $O\left(p^{4}\right)$ (including isospin breaking). To the order we are working, $\rho^{+}-\rho^{0}$ mass difference and $\rho-\omega$ mixing do not appear. Such higher-order effects (from the low energy point of view) are not necessarily negligible numerically (see, e.g., Ref. [1] $\left.{ }^{3}\right]$ ). They can be included as additional contributions to the factor $R_{\mathrm{IB}}(t)$.

To first order in isospin violation, this time including explicit photonic corrections, the form factor $f_{+}$is given by

$$
f_{+}(t, u)=1+2 H_{\pi^{0} \pi^{-}}(t)+H_{K^{0} K^{-}}(t)+f_{\text {loop }}^{\text {elm }}\left(u, M_{\gamma}\right)+f_{\text {local }}^{\text {elm }} .
$$

Compared to the form factor $F_{V}(t)$ in $(\overline{2} 2.11)$, the appropriate meson masses appear in the loop amplitude and there is an additional electromagnetic amplitude, containing both the photon loop diagrams and an associated local part. The electromagnetic amplitude depends on the second Dalitz variable $u=\left(P_{\tau}-p_{\pi^{-}}\right)^{2}$. The loop function $f_{\text {loop }}^{\text {elm }}\left(u, M_{\gamma}\right)$ encodes universal physics related to the Coulomb interaction between the $\tau$ and the charged pion, and therefore we pull it out in an overall term. Matching this low energy result to the resonance region, we are lead to write:

$$
\begin{aligned}
f_{+}(t, u) & =f_{+}(t)\left[1+f_{\text {loop }}^{\text {elm }}\left(u, M_{\gamma}\right)\right] \\
f_{+}(t) & =\frac{M_{\rho}^{2}}{M_{\rho}^{2}-t-i M_{\rho} \Gamma_{\rho}(t)} \exp \left[2 \operatorname{Re} \tilde{H}_{\pi^{0} \pi^{-}}(t)+\operatorname{Re} \tilde{H}_{K^{0} K^{-}}(t)\right]+f_{\text {local }}^{\text {elm }} .
\end{aligned}
$$

The resonance width $\Gamma_{\rho}(t)$ in $(\overline{2}-4)$ has to be calculated now with the appropriate $\pi^{-} \pi^{0}$ and $K^{-} K^{0}$ thresholds and phase space factors. The local contribution in $f_{+}$depends on three low-energy constants appearing in the chiral expansion. The bounds used for them [i] reflect in the uncertainty reported in our result (Fig. 戛 $(a)$, solid curves).

The photon loop amplitude $f_{\text {loop }}^{\text {elm }}\left(u, M_{\gamma}\right)$ is infrared divergent, depending on an artificial photon mass $M_{\gamma}$. This dependence is canceled by bremsstrahlung of soft photons making 

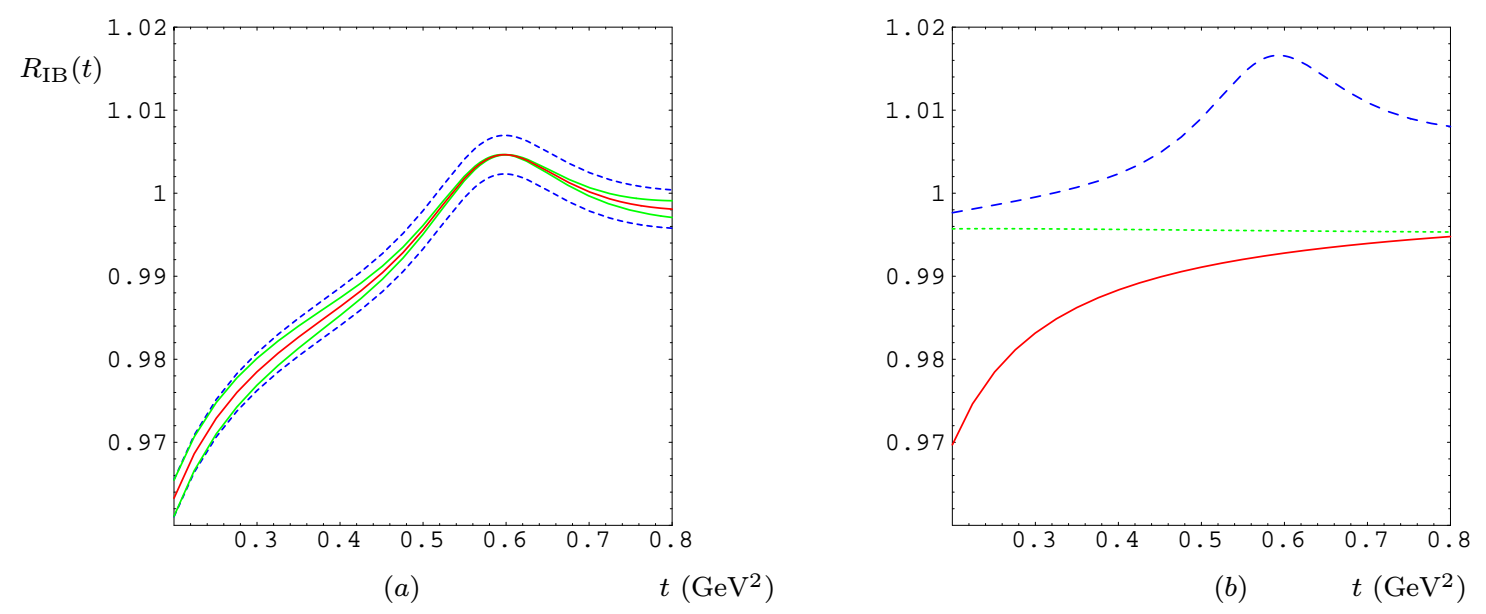

Figure 1: $(a)$ Correction factor $R_{\mathrm{IB}}(t)$ for isospin violation. The bands around the central curve correspond to the uncertainty in the low-energy constants (solid lines) and in the bremsstrahlung factor (dashed lines). (b) The separate factors defining $R_{\mathrm{IB}}(t)$ in Eq.(11.6i) are plotted as solid line for $\beta_{+-}^{3} / \beta_{0-}^{3}$, dashed line for $\left|F_{V}(t) / f_{+}(t)\right|^{2}$ and dotted line for $1 / G_{\mathrm{EM}}(t)$.

the decay distribution in $(t, u)$ infrared finite. The sum of real and virtual contributions produces a correction factor $\Delta(t, u)$ to the $(t, u)$ decay distribution. After averaging over the Dalitz variable $u$, this produces the term $G_{\mathrm{EM}}(t)$ in the decay distribution (1.3.3.). The precise form of $\Delta(t, u)$ (and $G_{\mathrm{EM}}(t)$ ) depends on the specific experimental setup. To the best of our knowledge, all $\tau$ decay experiments relevant here [5] apply bremsstrahlung corrections in the same (approximate) way [14] including only the leading term in the Low expansion. Assuming this prescription, we have then calculated the setup-independent part of $G_{\mathrm{EM}}(t)$, assigning to it an uncertainty of $\pm \alpha / \pi$ (due to neglect of sub-leading terms).

\section{Results and conclusions}

The results of our analysis are summarized in Figs. $\underline{1}_{1}^{1}(a),(b)$ where we plot the function $R_{\mathrm{IB}}(t)$ of Eq. (1) and its component factors for $0.2 \leq t \leq 0.8 \mathrm{GeV}^{2}$. We note that the dominant contribution at low $t$ is given by the kinematical term $\beta_{+-}^{3} / \beta_{0-}^{3}$ [1 ${ }_{i}^{1} 0_{1}^{1}$. Photonic corrections embodied in $G_{\mathrm{EM}}(t)$ reduce $R_{\mathrm{IB}}(t)$ in addition by about half a percent, largely independently of $t$. The form factor ratio $\left|F_{V}(t) / f_{+}(t)\right|^{2}$ is dominated by the width difference $\Gamma_{\rho^{+}}-\Gamma_{\rho^{0}}$. Since $R_{\mathrm{IB}}(t)$ is smaller than unity in most of the region under consideration $\left(4 M_{\pi}^{2} \leq t \leq 0.8 \mathrm{GeV}^{2}\right)$, isospin violation accounts for a sizable part of the systematic difference at low energies between $e^{+} e^{-}$and $\tau$ decay data (e.g., Ref. [i1 $\left.\overline{1}_{1}\right]$ ).

In order to quantify the impact of $R_{\mathrm{IB}}(t)$ on $a_{\mu}^{\text {vacpol }}$, we construct the following ratio:

$$
\mathcal{R}\left(t_{\max }\right)=\frac{\int_{4 M_{\pi}^{2}}^{t_{\max }} d t K(t) \sigma_{e^{+} e^{-} \rightarrow \pi^{+} \pi^{-}}^{0, \mathrm{CVC}}(t) R_{\mathrm{IB}}(t)}{\int_{4 M_{\pi}^{2}}^{t_{\max }} d t K(t) \sigma_{e^{+} e^{-} \rightarrow \pi^{+} \pi^{-}}^{0, \mathrm{CVC}}(t)},
$$

and report a few representative values of $\mathcal{R}\left(t_{\max }\right)$ in Table $\underline{11}_{1}$ 
Although the calculation is based on a lowenergy description of the standard model, we claim that the main features of our $R_{\mathrm{IB}}(t)$ are valid up to $t \simeq 0.8 \mathrm{GeV}^{2}$. Of the three factors in the definition (1.6) of $R_{\mathrm{IB}}(t)$, both the dominant phase space correction factor [i] $1 \overline{0}]$ and the photon loop effects are independent of the low-energy expansion. Finally, the main part of isospin violation in the form factor ratio $\left|F_{V}(t) / f_{+}(t)\right|^{2}$ occurs in the $\rho$-width difference $\Gamma_{\rho^{+}}-\Gamma_{\rho^{0}}$ and should therefore be reliable in

\begin{tabular}{|c||c|c|c|}
\hline$t_{\max }\left(\mathrm{GeV}^{2}\right)$ & 0.3 & 0.5 & 0.8 \\
\hline $\mathcal{R}\left(t_{\max }\right)$ & 0.949 & 0.974 & 0.988 \\
\hline
\end{tabular}

Table 1: Correction factor for $a_{\mu}^{\text {vacpol }}$ due to isospin violation (defined in Eq. (3.1.j) for some values of $t_{\max }$. An uncertainty of 0.002 - due to $G_{\mathrm{EM}}(t)$ - should be assigned to the reported values. This is also an upper bound for the uncertainty due to the low-energy constants (see Fig. $\prod_{1}^{1}(a)$ ). the vicinity of the resonance.

\section{References}

[1] V. Cirigliano, G. Ecker, H. Neufeld, Phys. Lett. B513 (2001) 361.

[2] M. Gourdin and E. de Rafael, Nucl. Phys. B10 (1969) 667.

[3] S. Narison, Phys. Lett. B513 (2001) 53.

[4] R. Alemany, M. Davier and A. Höcker, Eur. Phys. J. C2 (1998) 123;

M. Davier and A. Höcker, Phys. Lett. B435 (1998) 427.

[5] R. Barate et al. (ALEPH), Eur. Phys. J. C4 (1998) 409;

K. Ackerstaff et al. (OPAL), Eur. Phys. J. C7 (1999) 571;

S. Anderson et al. (CLEO), Phys. Rev. D61 (2000) 112002.

[6] H.N. Brown et al. (BNL-E821), Phys. Rev. Lett. 86 (2001) 2227.

[7] S. Weinberg, Physica 96A (1979) 327;

J. Gasser and H. Leutwyler, Ann. Phys. 158 (1984) 142;

J. Gasser and H. Leutwyler, Nucl. Phys. B250 (1985) 465.

[8] R. Urech, Nucl. Phys. B433 (1995) 234;

H. Neufeld and H. Rupertsberger, Z. Phys. C68 (1995) 91; ibid. C71 (1996) 131;

M. Knecht, H. Neufeld, H. Rupertsberger and P. Talavera, Eur. Phys. J. C12 (2000) 469.

[9] W.J. Marciano and A. Sirlin, Phys. Rev. Lett. 61 (1988) 1815; ibid. 71 (1993) 3629.

[10] H. Czyz and J.H. Kühn, Eur. Phys. J. C18 (2001) 497.

[11] J. Gasser and H. Leutwyler, Nucl. Phys. B250 (1985) 517.

[12] F. Guerrero and A. Pich, Phys. Lett. B412 (1997) 382;

D. Gómez Dumm, A. Pich and J. Portolés, Phys. Rev. D62 (2000) 054014.

[13] K. Maltman, Phys. Rev. D58 (1998) 014008.

[14] E. Barberio and Z. Wạs, Comp. Phys. Comm. 79 (1994) 291.

[15] S.I. Eidelman and V.N. Ivanchenko, Nucl. Phys. B (Proc. Suppl.) 76 (1999) 319. 